\title{
Introduction
}

\section{Disability evaluation}

I consider it an honor to serve as guest editor for this issue on Disability Evaluation. The notion that disability evaluation is an integral part of the practice of medicine is presently a hot issue, and I commend the editor of the Journal of Back and Musculoskeletal Rehabilitation, Dr. Karen Rucker, for bringing this important issue to your attention. In choosing the authors for this issue, I have attempted to select individuals who have had extensive experience in dealing with the selected topics and provide a balance of subject matter in both disability evaluation and impairment rating.

Saving lives is a common occurrence for trauma surgeons. As physicians, we have all been trained to respond quickly and efficiently in emergency situations and contribute directly or indirectly to salvaging mutilated bodies and limbs. Following lengthy recoveries, these patients face long-term management of chronic disabilities as a result of their trauma. A large number of disabled individuals do not have such a dramatic history but nevertheless face equally long periods of rehabilitation.

The greatest challenge to rehabilitation personnel is giving the reconstructed and disabled patient the will to become a whole human being again; to become a person who can interact normally with family members, friends, co-workers, and the community in general. The task is frequently more difficult than the actual surgical reconstruction.

Once such a traumatized patient is deemed well enough to begin such activities as feeding themselves, getting out of bed, and tending to personal hygiene and self-care, we call upon re- habilitation personnel to take over the care and management of what we consider reconstructed successes - frequently without realizing that this is just the beginning of an arduous and complex sequence of upcoming events.

The psychological effects of severe trauma may well eventually be more disabling than the actual effects of the trauma itself. Our modern lifestyle is not structured to accommodate the long-lasting effects of severe trauma. The patient with such minor injuries as a simple fracture of a finger may display significant frustration when he finds that the injured finger is still significantly swollen and tender 3-4 weeks later and interferes with his participation in a bowling league competition. Yet, this patient can be assured that $8-10$ months from now he will likely have forgotten which finger had been involved. The same individual may experience employer dissatisfaction that his piecework production has been affected by the non-work related injury.

Our social-economic environment does not tolerate disability well. Individually and collectively, we have been conditioned to expect that accidents and ensuing disabilities occur to others but not to ourselves. We are ill-prepared psychologically for the shock of a long-term disability or even less so for one that leaves a permanent impairment especially if that impairment is visible or perceived to be visible by the individual.

Dealing with the rehabilitation of disabled individuals and those who remain with physical or cognitive impairments need obvious attention and 
management of the physical problem. Simultaneously, psychological evaluation, detection, and early management of any psychological barriers to rehabilitation must be carried out if success is to be expected. Early involvement of family support and education of family members as to what they can expect from the disabled individual once he has returned to the family circle, may facilitate the rehabilitation process. The loss of self-esteem, tendency to depression, temper flare-ups, impatience, pressures concerning the ability to return to work and at what level, loss of confidence in physical performance or memory deficits, all need to be dealt with to ensure the best possible result. While doing this, we need to deal with issues such as the belief that a physical limitation precludes the ability to hold onto a job or that a physical defect makes the individual less of a man or woman. We must be aware of the tendency of disabled individuals to try to isolate themselves from the rest of society and, in particular, from previous friends and acquaintances with whom he has previously worked.

Therein may lie the weakness of the concept of maximum medical improvement which is gener- ally accepted as the time when the individual has achieved maximum physical improvement and should require no further medical treatments. Indeed, what we should be looking for primarily is maximum functional improvement which may not be reached until the individual becomes integrated back into his family, social, and work environment. Often there is a large discrepancy between maximum physical improvement and maximum functional improvement. This is due to failure of integration by the patient into his prior work environment or into a new work environment as resumption of previous activities of daily living become more challenging.

The scope of this issue on Disability Evaluation is to provide informative articles from various aspects of disability evaluation and impairment rating, and to hopefully provide a well-rounded symposium on this provocative subject matter.

Leonard D. Emond, MD, FAADEP, FICS Past President, $A A D E P$ President, New England Disability Evaluations Manchester, New Hampshire 